\title{
The Method for Risk Assessment of SSR Caused by Doubly-Fed Wind Farms
}

\author{
Xiao Yu, Guofei Lu, Wuhui Chen and Danhui Wang \\ School of Electrical and Information Engineering, Jiangsu University, Zhenjiang, China
}

\begin{abstract}
The existing method for investigating the subsynchronous resonance (SSR) caused by wind power generation is mainly aimed at a deterministic condition. In order to analyse the impact of uncertain factors on SSR in wind farms, this paper defines the risk matrix and risk index, and develops a SSR-oriented risk assessment method of using probabilistic collocation method (PCM). Considering the uncertain of wind speeds, the proposed method is used to assess the SSR risk of a wind farm. The results show that under the same wind speed distribution, the higher the series compensation level in the system is, the greater the SSR risk of the system could be; under the same series compensation level, the SSR risks caused by different wind speed distribution are different, and the system in the areas with lower average wind speed obtains greater SSR risk.
\end{abstract}

\section{$1 \quad$ INTRODUCTION}

Large-scale development and utilization of wind energy has become an important part of the energy strategy of many nations (Liserre et al, 2011). Since wind energy resources are generally far from the load centre, the series compensation technique is widely adopted as an efficient measure to improve the capability of long-distance transmission of wind power (Mohammadpour et al, 2014). However, there exists the potential risk of subsynchronous resonance (SSR) for such a wind farm when it is connected to series-compensated networks (Ostadi et al, 2009). In October 2009, at two doubly-fed induction-generator (DFIG) based wind farms in Southern Texas, USA, a forced outage left the wind farms radially connected to the main power grid. A serious SSR incident occurred and destroyed a large number of wind turbines (Adams et al, 2012).

Much research work has been done to analyse the stability of this DFIG-related SSR (Fan et al, 2011). However, in most existing work, SSR was investigated under deterministic operating conditions, without considering the uncertainty of some key factors, for instance, the stochastic wind speed. Thus, it is difficult to fully understand the risk of SSR and take reasonable countermeasures to address the issue.

To fully consider the impact of uncertainties, especially the stochastic wind speed, this paper defines the risk matrix and risk index, and develops a SSR-oriented risk assessment method of using probabilistic collocation method (PCM). The SSR risk of a wind farm with seriescompensated lines is assessed with this method under all the possible wind speed.

\section{PROBABILISTIC ASSESSMENT}

When the power system is in operating mode $i$, the occurrence of an emergency event $j$ could make the system state transit to $x_{i j}$. The security risk in this state can be defined as the product of the probability of the event $P\left(x_{i j}\right)$ and the resulting impact (or loss) $S\left(x_{i j}\right)$ :

$$
R_{i j}=P\left(x_{i j}\right) \times S\left(x_{i j}\right)
$$

The relationship between the risk-based safety assessment and deterministic safety assessment can be shown in Figure 1. The area surrounded by solid line is a safe area determined by deterministic research methods. The dashed lines are security zone boundaries with two different risks given by risk-based security assessment method, with a risk of $5 \%$ and $10 \%$, respectively. According to the risk security zone, the deterministic security zone can be divided into three areas: Area A, Area $\mathrm{B}$, and Area $\mathrm{C}$. The operation mode has less than $5 \%$ risk in Area A, which is too conservative. The system can take greater risk. The operation mode has more than $10 \%$ risk in Area $\mathrm{C}$, which is overly optimistic when judged by deterministic assessment method under this vulnerable state. The difference between the risk assessment method and the deterministic assessment method is that the risk assessment method takes into account the randomness and variation of many parameters of the system under various operating conditions. The risk index combines the probability with the severity of the accident, which can reflect the security situation of the power system generally.

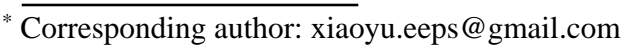




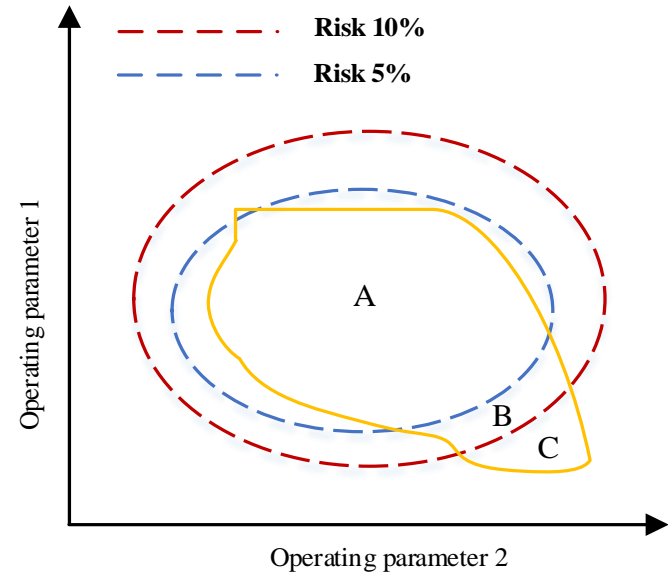

Figure 1: Stability regions under the deterministic method and risk-based method.

The SSR issues of wind farms involve many uncertainties such as wind speed, system parameters, operation modes and fault types. It is necessary to use risk assessment method to analyze the SSR risk with actual wind power grid-connected models.

\section{PROBABILISTIC COLLOCATION METHOD}

Probabilistic collocation method (PCM) essentially creates a high-order polynomial model to approximate the functional relationship between the system random output and uncertain input parameters (Tatang, 1995). $x$ is a single uncertain parameter (or fault event) of the power system. Under the action of the $x$, it is assumed that the random output $Y=g(x)$ of the system is a function of the $x$. The $Y$ can be approximated by a polynomial model $\bar{Y}$ :

$$
\bar{Y}=\bar{g}(x)=\sum_{i=0}^{n-1} b_{i} H_{i}(x)=b_{0} H_{0}(x)+\ldots+b_{n-1} H_{n-1}(x)
$$

Where $\mathrm{i}=0, \ldots, \mathrm{n}-1 ; \mathrm{Hi}(\mathrm{x})$ is an $\mathrm{i}$-order polynomial about x. All Hi(x) constitute a polynomial sequence $H ; b_{i}$ is a PCM model coefficient and $n-1$ is the order of PCM model (Hockenberry et al, 2004). Based on the probability density function (PDF) of the uncertain input parameters, orthogonal polynomials and Gaussian integral theory (Preece et al, 2013 ) are used to solve the polynomials and collacations of the PCM models respectively. Therefore, the orthogonal polynomial theory and Gaussian integration theory will be introduced before presenting the PCM model solution process.

\subsection{ORTHOGONAL POLYNOMIAL THEORY}

The inner product of the polynomial is defined in Equation (3):

$$
\langle L(x), H(x)\rangle=\int_{\alpha} f(x) L(x) H(x) d x
$$

Where: $\alpha$ is the distribution area of the parameter $x ; f(x)$ is the non-negative weight function in the defined $\alpha$ region; $L(x)$ and $H(x)$ are two polynomials. In the PCM, $f(x)$ is the PDF of the parameter $x$.
As shown in Equation (4), if the integral of the two polynomials with the weight function $f(x)$ is 0 , the two polynomials are orthogonal. Assuming that the different order polynomials in the polynomial sequence $H$ are pairwise orthogonal, the $H$ is defined as an orthogonal polynomial sequence (Davis et al, 1975).

$$
\left\langle H_{j}, H_{k}\right\rangle=\int_{\alpha} f(x) H_{j}(x) H_{k}(x) d x= \begin{cases}0 & j \neq k \\ A>0 & j=k\end{cases}
$$

Where $H_{j}$ and $H_{k}$ are the $j$-order and $k$-order polynomial in the polynomial sequence $H ; j, k=0,1$. Given the $\operatorname{PDF} f(x)$ for any uncertain parameter $x$ and defining $H_{-1}(x)=0$, $H_{0}(x)=1$, The orthogonal polynomials $H_{i}(x)$ can be obtained. Here, we use $H_{0}(x)=1$ and $H_{1}(x)=x-c_{1}$ as the starting point to generate a set of orthogonal polynomials through the standard Gramschmidt orthogonal transformation:

$$
\begin{gathered}
H_{i}(x)=\left(x-c_{i}\right) H_{i-1}(x)-d_{i} H_{i-2}(x) \\
c_{i}=\frac{\left\langle x H_{i-1}, H_{i-1}\right\rangle}{\left\langle H_{i-1}, H_{i-1}\right\rangle} \\
d_{i}=\frac{\left\langle H_{i-1}, H_{i-1}\right\rangle}{\left\langle H_{i-2}, H_{i-2}\right\rangle}
\end{gathered}
$$

Where $H_{i}(x), H_{i-1}(x)$ and $H_{i-2}(x)$ are the orthogonal polynomials about the wind speed $x$, and $c_{1}$ is the average value of the parameter $x$.

Each orthogonal polynomial obtained by Equation (5) has $i$ roots, and these roots are all in the $\alpha$ domain. These roots will be used to solve PCM model parameters.

\subsection{GAUSSIAN INTEGRATION THEORY}

The Gaussian integration method can be expressed as follows:

$$
\int_{\alpha} f(x) g(x) d x=\sum_{i=0}^{n-1} \mathrm{~A}_{i} g\left(x_{i}\right)
$$

Where: $g(x)$ is a polynomial and $f(x)$ is a weight function. If $f(x)$ is a PDF, the integral of Equation (6) is the mathematical expectation of polynomial $g(x) . i=0, n-1$. $\mathrm{A}_{i}$ is a constant that depends only on the $f(x)$ rather than $g(x)$; $x_{i}$ is a constant within the integral region $\alpha$. Appropriate choose of $\mathrm{A}_{i}$ and $x_{i}$ can make Equation (6) exact for $g(x)$, which is called Gaussian point. The reference (Davis et al, 1975) gives the Gaussian point selection theorem that the zero point of the orthogonal polynomial $H_{n}(x)$ in the region $\alpha$ is taken as a Gaussian point. The sequence $H$ can be regarded as a set of orthonormal bases, because the polynomials in the $H$ are pairwise orthogonal.

Since $H_{n}(x) H_{n-1}(x), H_{n}(x) H_{0}(x), H_{n-1}(x), H_{0}(x)$ are $2 n-1$ linearly independent polynomials (but not necessarily orthogonal), the $2 n-1$ order $g(x)$ can be expressed with $2 n$ 1 linearly independent polynomials in the following form:

$$
\begin{gathered}
g(x)=H_{n}(x)\left(a_{n-1} H_{n-1}(x)+\ldots+a_{0} H_{0}(x)\right)+ \\
b_{n-1} H_{n-1}(x)+\ldots+b_{0} H_{0}(x)
\end{gathered}
$$

Where: $a_{0}, a_{n-1}, b_{0}, b_{n-1}$ are constant. According to Equation (4), the integral of the $2 n$-1 order polynomial $g(x)$ can be written as follows:

$$
\int_{\alpha} f(x) g(x) d x=b_{0} \int_{\alpha} f(x) H_{0}(x) d x
$$


Substituting the $n$ roots $x_{i}$ of the orthogonal polynomials $H_{n}(x) \quad(i=0, n-1)$ (Gaussian points) into Equation (7), a system of linear equations is given :

$$
\left[\begin{array}{c}
g\left(x_{0}\right) \\
\vdots \\
g\left(x_{n-1}\right)
\end{array}\right]=\left[\begin{array}{ccc}
H_{n-1}\left(x_{0}\right) & \cdots & H_{0}\left(x_{0}\right) \\
\vdots & \ddots & \vdots \\
H_{n-1}\left(x_{n-1}\right) & \cdots & H_{0}\left(x_{n-1}\right)
\end{array}\right]\left[\begin{array}{c}
b_{n-1} \\
\vdots \\
b_{0}
\end{array}\right]
$$

Where $g\left(x_{i}\right)$ is the value of $g(x)$ at $x_{i}$, and $H_{i}\left(x_{i}\right)$ is the value of the orthogonal polynomial at $x_{i}$. Inverse of equation (8) is as follows:

$$
\left[\begin{array}{c}
b_{n-1} \\
\vdots \\
b_{0}
\end{array}\right]=\left[\begin{array}{ccc}
H_{n-1}\left(x_{0}\right) & \cdots & H_{0}\left(x_{0}\right) \\
\vdots & \ddots & \vdots \\
H_{n-1}\left(x_{n-1}\right) & \cdots & H_{0}\left(x_{n-1}\right)
\end{array}\right]^{-1}\left[\begin{array}{c}
g\left(x_{0}\right) \\
\vdots \\
g\left(x_{n-1}\right)
\end{array}\right]
$$

Assuming that $H_{0}(x)=1$ and $f(x)$ is a PDF, the expression of $b_{0}$ is given with reference to Equation (9):

$$
b_{0}=\int_{\alpha} f(x) g(x) d x=\sum_{i=0}^{n-1} \mathrm{~A}_{i} g\left(x_{i}\right)
$$

As shown in Equation (9), $A_{i}$ can be obtained by the last row of the matrix and depends only on the orthogonal polynomial $H_{i}(x)$. Since $H_{i}(x)$ and $x_{i}$ depend only on the weight function $f(x)$, both the $\mathrm{A}_{i}$ and $x_{i}$ are independent of $g(x)$. In the PCM, this independence is equivalent to the independence between a small number of collocation points and the random output function. Therefore, it is sufficient to get model coefficient by simulating a set of Gaussian points for a PCM polynomial $g(x)$ with an order less than $2 n-1$.

According to Gaussian integration theory, $x$ is the system input parameter, $f(x)$ is the PDF of parameter $x$ and $g(x)$ is the random output of the system. The mathematical expectation of the PCM model is consistent with the results obtained with a set of Gaussian points. The statistical properties of the PCM model can reflect the characteristics of the random output function $g(x)$ of the system. Therefore, it is proved that Equation (2) is feasible.

\section{THE QUANTIFICATION OF SSR RISK}

According to Equation (1), the SSR risk is the product of the probability and the severity of SSR. Generally, to quantify the SSR risk, the SSR risk is indicated by a noncontinuous risk matrix and the SSR severity is indicated by a continuous risk function.

\subsection{RISK MATRIX}

The SSR risk can be characterized by amplitude and settling time of the oscillation. The settling time depends on the damping factor. It is defined as the duration in which the amplitude of the oscillation attenuates to $5 \%$ of its initial value, or

$$
T_{s}=\frac{\ln (0.05)}{\sigma}=\frac{-3.00}{\sigma}
$$

Table 1: Risk matrix for analysis of SSR in wind power system.

\begin{tabular}{ccccc}
\hline \hline \multirow{2}{*}{ Probability } & \multicolumn{4}{c}{ Settling Time } \\
\cline { 2 - 5 } $0 \%$ & Unstable & $>8 \mathrm{~s}$ & $3-8 \mathrm{~s}$ & $<3 \mathrm{~s}$ \\
$(0-1 \%]$ & Safe & Safe & Safe & Safe \\
$(1-10 \%]$ & Sevtable & Safe & Safe & Safe \\
$(10-30 \%]$ & Acceptable & Safe & Safe \\
$(30-50 \%]$ & Severe & Acceptable & Safe & Safe \\
$(50-100 \%]$ & Severe & Severe & Acceptable & Safe \\
\hline \hline
\end{tabular}

Then, a risk matrix is specified to characterize SSR security. As shown in Table 1, the matrix, with its row and its column denoting the settling time and the occurrence probability, in their crossing points offers a qualitative description of system security in terms of SSR risk. The 3s and $8 \mathrm{~s}$ are chosen as the lines of settling time, which correspond to SSR damping $\sigma=-1$ and $\sigma=-0.375$ respectively.

\subsection{RISK INDEX}

A continuous severity function $S(\sigma)$ is defined in Equation (11) to calculate the risk index $R . P(\sigma)$ is the PDF of the SSR modal damping under stochastic wind speed. In this paper, a step function is taken as the severity function according to the actual situation: The longer the settling time is, the greater the risk will be. The risk $R$ can be defined as follows:

$$
R=\int P(\sigma) * S(\sigma) d \sigma
$$

where

$$
S(\sigma)=\left\{\begin{array}{lc}
0 & \sigma<-1 \\
0.1 & -1 \leq \sigma<-0.375 \\
0.2 & -0.375 \leq \sigma<0 \\
1 & \sigma \geq 0
\end{array}\right.
$$

\subsection{SSR RISK ASSESSMENT METHOD CONSIDERING STOCHASTIC WIND SPEED}

The PCM is used to quantify the SSR risk in a DFIG wind farm with series compensation by defining risk matrix and risk index. Assuming the stochastic wind speed $x$ as the system uncertain input parameter and SSR damping $\sigma(x)$ as the output of the system, the SSR risk assessment method considering stochastic wind speed is as follows:

(1) Select the PDF $f(x)$ of the stochastic wind speed.

(2) Obtain orthogonal polynomials $H_{i}(x)(i=0, n)$ by Equation (5).

(3) Calculate $n$ roots $x_{i}$ of orthogonal polynomials $H_{n}(x)$ and take them as $n$ collocation points of wind speed.

(4) Take Prony algorithm to analyse the SSR damping $\sigma\left(x_{i}\right)$ at collocation point $x_{i}$ when the system suffers from SSR.

(5) Use Equations (2), (8) and (9) to obtain the expression $\sigma(x)$ between wind power system SSR damping and uncertain parameter wind speed $x$.

(6) Use the Monte Carlo sampling technique to take 5,000 random wind speeds, substitute into $\sigma(x)$ to calculate the corresponding damping and plot the damping probability density curve in MATLAB. 
(7) Assess the SSR risk of the DFIG wind farm with series compensation through the risk matrix defined in Table 1 and the risk index defined in Equation (11).

\section{CASE STUDY}

\subsection{MODELLING}

The models based on practical scenes are necessary when analysing the SSR induced by DFIG wind farms with series compensation. In this paper, the power is delivered from DFIG wind farm to the remote load centre through dual loop $345 \mathrm{kV}$ lines. Both lines are equipped with series compensation. The wind farm consists of 50 DFIG wind turbines, whose single rated capacity is $2 \mathrm{MW}$ and rated voltage is $690 \mathrm{~V}$. The voltage is stepped up to $26 \mathrm{kV}$ and then connected to the grid through the $26 / 345 \mathrm{kV}$ main transformer of the wind farm. The parameters of a DFIG are as follows: the stator resistance $R_{s}=0.015$ p.u., the rotor resistance $\mathrm{R}_{\mathrm{r}}=0.0507$ p.u., the stator leakage inductance $\mathrm{L}_{\mathrm{s}}=0.091$ p.u., the rotor leakage inductance $\mathrm{L}_{\mathrm{r}}=0.05$ p.u., the magnetizing inductance $\mathrm{L}_{\mathrm{m}}=2$ p.u. When the operating states of all wind turbines in the wind farm are the same or with little differences, the entire wind farm can be represented by a single equivalent model (Fernández et al, 2008). The equivalent circuit of the wind farm with series compensated lines is shown in Figure 2.

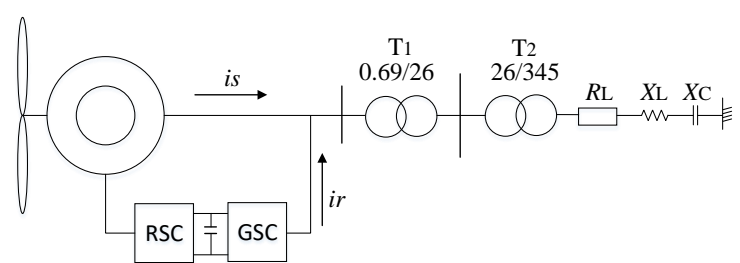

Figure 2: Equivalent circuit of the doubly fed wind farm connected to a series-compensated system.

As shown in Figure 2, $\mathrm{T}_{1}$ and $\mathrm{T}_{2}$ are step-up transformers; $i_{s}$ is stator output current; $i_{r}$ is rotor output current through the converter; $R_{L}$ is transmission line resistance; $X_{L}$ is line reactance; $X_{C}$ is series capacitive reactance; GSC and RSC are grid-side converters and rotor-side converters. The simulation model based on Figure 2 is established in the PSCAD. The Prony algorithm is used to extract the SSR modal damping of the system.

There are several existing models to describe the probabilistic distribution of wind speed, for instance, Rayleigh distribution (Manwell et al, 2006), Weibull distribution (Jangamshetti et al, 1999) and Lognormal distribution (Garcia et al, 1998). The Weibull distribution is the most widely used.

The PDF of Weibull distribution are expressed as:

$$
f(v)=\left(\frac{k}{c}\right)\left(\frac{v}{c}\right)^{k-1} \exp \left[-\left(\frac{v}{c}\right)^{k}\right], v \geq 0
$$

Where $v$ is the speed of wind in $\mathrm{m} / \mathrm{s} ; c, k$ are the scale parameter and the shape parameter, respectively, which can be obtained by solving the following equation:

$$
\begin{aligned}
& \mu=c \Gamma\left(1+\mathrm{k}^{-1}\right) \\
& \delta^{2}=c^{2} \Gamma\left(1+2 \mathrm{k}^{-1}\right)-\mu^{2} \\
& \Gamma(x)=\int_{0}^{\infty} \mu^{x-1} \exp (-\mu) d \mu, \mu>0
\end{aligned}
$$

The severity of the oscillations are different with the change of the series compensation level under the same wind speed. Therefore, when the compensation levels are $50 \%, 60 \%$ and $70 \%$, the corresponding system resonant frequencies are $20 \mathrm{~Hz}, 23 \mathrm{~Hz}$ and $25 \mathrm{~Hz}$, respectively. The SSR risk will be calculated under these series compensation levels.

\subsection{SSR RISK ANALYSIS}

In this case, the input parameter is the wind speed of the wind farm and the output is the SSR modal damping $\sigma(x)$ at the corresponding wind speed. The average value of the wind speed $\mu$ is 7 and the standard deviation $\delta$ is 5.3. With reference to Equations (5) and (13), the 3-order orthogonal polynomial of the wind speed is calculated. The roots of $H_{3}(x)$ are $x_{1}=3.00 \mathrm{~m} / \mathrm{s}, x_{2}=8.58 \mathrm{~m} / \mathrm{s}, x_{3}=16.01 \mathrm{~m} / \mathrm{s}$, which are taken as collocations. Table 2 shows the subsynchronous modal damping for different collocations.

Table 2: Analysis of subsynchronous modal damping for different collocations.

\begin{tabular}{cc|ccc}
\hline \multirow{2}{*}{\multicolumn{2}{c|}{ Damping }} & Level 1 & Level 2 & Level 3 \\
\cline { 2 - 5 } & & $50 \%$ & $60 \%$ & $70 \%$ \\
\hline \multirow{4}{*}{$x$} & 3.0 & -0.4212 & -0.2239 & 0.1401 \\
& 8.6 & -0.7718 & -0.4918 & -0.3710 \\
& 16.0 & -1.3636 & -1.1321 & -0.9375 \\
\hline
\end{tabular}

Approximate expressions of PCM model under three different series compensation levels can be obtained from Equations (2), (8), and (9):

Level 1:

$$
\sigma=-0.0093 x^{2}+0.0450 x-0.4728
$$

Level 2:

$$
\sigma=-0.0121 x^{2}+0.0919 x-0.3897
$$

Level 3:

$$
\sigma=-0.0056 x^{2}-0.0266 x+0.2683
$$

5,000 stochastic wind speed points sampled by Monte Carlo technique are substituted into Equations (14), (15) and (16) to calculate damping under three series compensation levels. Since the cut-in wind speed is $3 \mathrm{~m} / \mathrm{s}$, the rated wind speed is $12.5 \mathrm{~m} / \mathrm{s}$ and the cut-out wind speed is $20 \mathrm{~m} / \mathrm{s}$, the damping data whose wind speeds are less than $3 \mathrm{~m} / \mathrm{s}$ or greater than $20 \mathrm{~m} / \mathrm{s}$ should be deleted. Figures 3-5 are the probability density curves of the SSR damping at $50 \%, 60 \%$ and $70 \%$ series compensation levels by the smooth core density estimation (Deveaux, 2004). 


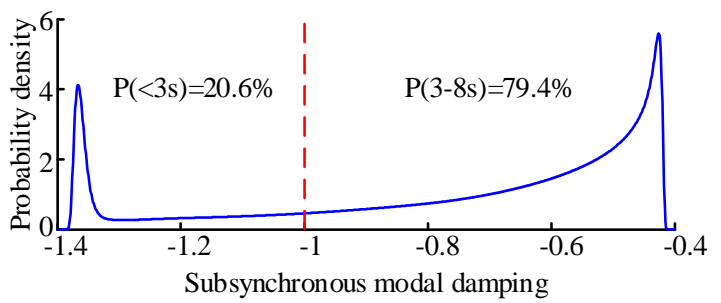

Figure 3: Probability density of subsynchronous modal damping with $50 \%$ series compensation.

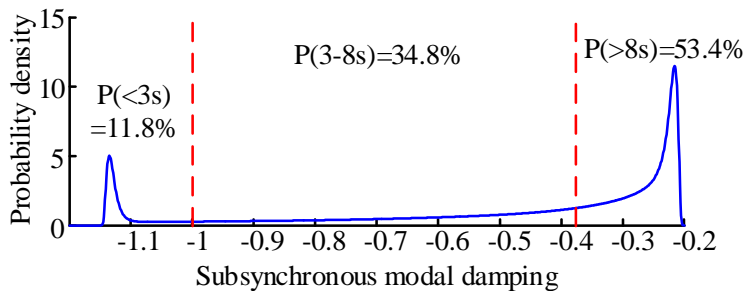

Figure 4: Probability density of subsynchronous modal damping with $60 \%$ series compensation.

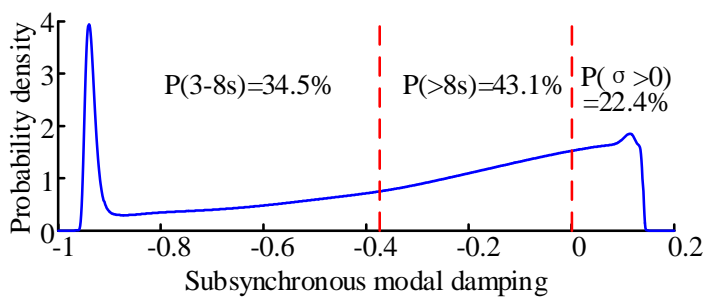

Figure 5: Probability density of subsynchronous modal damping with $70 \%$ series compensation.

As shown in Figures 3-5, the distributions of subsynchronous modal damping are different under different series compensation levels when SSR occur at stochastic wind speeds in this area. Referring to the risk matrix defined in Table 1, it can be concluded that the probability of settling time between $3 \mathrm{~s}$ and $8 \mathrm{~s}$ is $79.4 \%$ when the compensation level is $50 \%$. Although it is not within the safety range, it does not has serious damages to system security, so it is acceptable. When the series compensation level is $60 \%$, the probability that the settling time within $3 \mathrm{~s}$ and between $3-8 \mathrm{~s}$ is smaller than that of $50 \%$ series compensation level. Also, the probability of the settling time greater than $8 \mathrm{~s}$ is $53.4 \%$. It can be known that the SSR under this series compensation level is harmful to the system from the risk matrix, which is very dangerous at stochastic wind speeds. When series compensation level is $70 \%$, it can be seen from Figure 5 that the oscillation is diverging and the probability of the synchronous modal damping $\sigma$ greater than zero is $22.4 \%$. This operation mode is serious enough to undermine the safety and stability of the system. This condition must be avoided in practical operation. From the perspective of probability, it can be seen that, for the SSR induced by double-fed wind farms and series compensation, the greater the series compensation level, the greater damage to the system. Therefore, it is of great practical significance to properly select the suitable compensation to ensure the stability of the system when improve the transmission capacity.

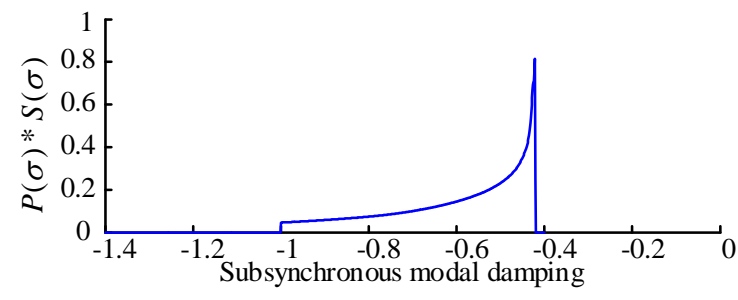

Figure 6: Evaluation of $\mathrm{P}(\sigma)^{*} \mathrm{~S}(\sigma)$ with $5.44 \%$ series

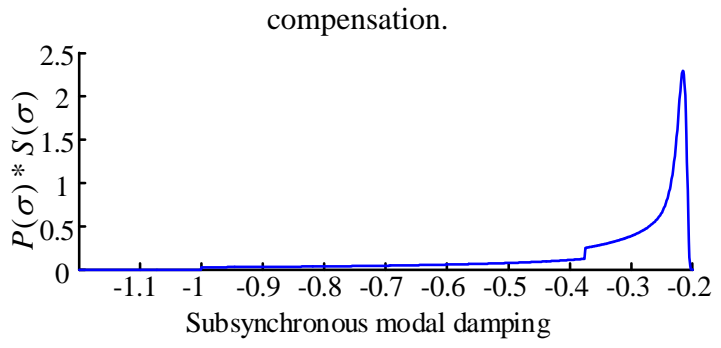

Figure 7: Evaluation of $P(\sigma) * S(\sigma)$ with $6.25 \%$ series compensation.

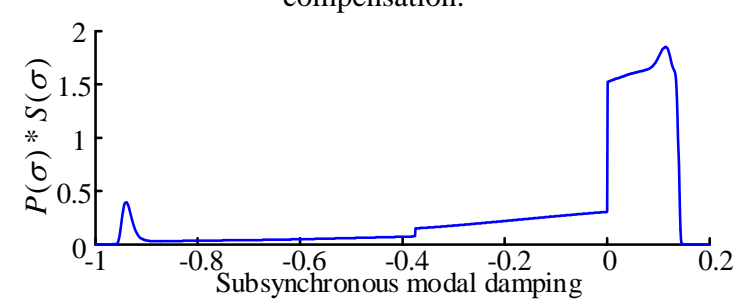

Figure 8: Evaluation of $\mathrm{P}(\sigma)^{*} \mathrm{~S}(\sigma)$ with $70 \%$ series compensation.

Figures 6-8 show the SSR analysis using the risk index under the $50 \%, 60 \%$ and $70 \%$ series compensation levels. The risks under three series compensation levels calculated by Equation (11) are 0.079, 0.142 and 0.345 , respectively. Obviously, the SSR risks at stochastic wind speeds exceed $10 \%$ under $60 \%$ and $70 \%$ series compensation levels, which seriously affect the reliability and stability of the power transmission. It is illustrated that the SSR risk increases with the increase of series compensation level.

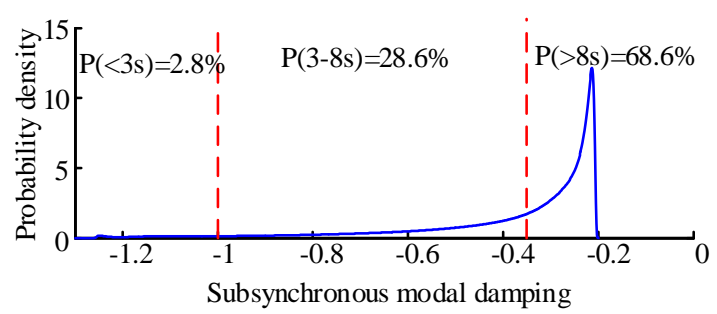

Figure 9: Probability density of modal damping with $60 \%$ series compensation $(\mathrm{k}=1.95)$.

Figure 9 shows the probability density curve of SSR damping under $60 \%$ series compensation level with $\mu=5, \delta=2.7, k=1.95$ and $c=5.64$. It can be seen that the probability that the settling time is greater than $8 \mathrm{~s}$ under this distribution coefficient is obviously greater than the probability under the wind speed distribution $k=2$ and $c=7.90$ at the same compensation level. The risk is calculated to be 0.166 , also apparently greater than 0.142 . From the perspective of risk matrix and risk index, it is shown that, for the SSR in different areas, the risk is not the same with different wind speed distribution and the areas with smaller average wind speed have greater risk under the same system conditions. 


\subsection{The Advantages of Probabilistic Safety Risk Assessment}

As it can be seen from Figures 4 and 7 that, with the wind speed distribution $k=2$ and $c=7.90$, the subsynchronous modal damping for each wind speed is below -0.2 , which means no divergence in the oscillation. Applying the deterministic method leads to the conclusion that wind power systems are safe to operate at $60 \%$ series compensation level. However, applying the risk assessment method, it can be seen that the probability of subsynchronous modal damping between -1 and -0.2 is $53.4 \%$ and the probability of settling time between $3 \mathrm{~s}$ and $8 \mathrm{~s}$ is $14.2 \%$, which corresponds to the Area $\mathrm{C}$ shown in Figure 1. It is shown that the risk assessment method can reflect the security situation of the power system more comprehensively.

\section{CONCLUSIONS}

This paper proposes a risk assessment method for the SSR induced by double-fed wind farms with series compensated lines. This method is applied to analyse the SSR risk considering stochastic wind speed. The conclusions are as follows:

(1) Under the same wind speed, the higher series compensation level can result in longer settle time, greater risk index and more serious damage to the system.

(2) Under the same series compensation level, different wind speed distributions result in different risks. In regions where the average wind speed is lower, the risk is greater than it in the regions with higher average speed.

(3) Compared with traditional deterministic method, the risk assessment method can reflect the security situation of the power system more comprehensively.

\section{ACKNOWLEDGEMENTS}

This research is supported by Open Fund of State Key Laboratory of Operation and Control of Renewable Energy \& Storage Systems (China Electric Power Research Institute)

\section{REFERENCES}

1. Adams, J., Carter, C., \& Huang, S. H. (2012). ERCOT experience with Sub-synchronous Control Interaction and proposed remediation. Transmission and Distribution Conference and Exposition (Vol.52, pp.1-5). IEEE.

2. Davis, P. J., \& Rabinowitz, P. (1975). Methods of numerical integration. Academic Press.

3. Deveaux, R. (2004). Applied smoothing techniques for data analysis. Technometrics, 41(3), 263-263.

4. Fan, L., Zhu, C., Miao, Z., \& Hu, M. (2011). Modal analysis of a dfig-based wind farm interfaced with a series compensated network. IEEE Transactions on Energy Conversion, 26(4), 1010-1020.
5. Fernández, L. M., Jurado, F., \& Saenz, J. R. (2008). Aggregated dynamic model for wind farms with doubly fed induction generator wind turbines. Renewable Energy, 33(1), 129-140.

6. Garcia, A., Torres, J. L., Prieto, E., \& Ade, F. (1998). Fitting wind speed distributions: a case study. Solar Energy, 62(2), 139-144.

7. Hockenberry, J. R., \& Lesieutre, B. C. (2004). Evaluation of uncertainty in dynamic simulations of power system models: the probabilistic collocation method. IEEE Transactions on Power Systems, 19(3), 1483-1491.

8. Jangamshetti, S. H., \& Rau, V. G. (1999). Site matching of wind turbine generators: a case study. IEEE Transactions on Energy Conversion, 14(4), 1537-1543.

9. Liserre, M., Cardenas, R., Molinas, M., \& Rodriguez, J. (2011). Overview of multi-mw wind turbines and wind parks. IEEE Transactions on Industrial Electronics, 58(4), 1081-1095.

10. Manwell, J. F., Mcgowan, J. G., \& Rogers, A. L. (2006). Wind Energy Explained: Theory, Design and Application. john Wiley \& Sons.

11. Mohammadpour, H. A., \& Santi, E. (2014). Subsynchronous resonance analysis in DFIG-based wind farms: Definitions and problem identification — Part I. Energy Conversion Congress and Exposition (pp.812-819). IEEE.

12. Ostadi, A., Yazdani, A., \& Varma, R. K. (2009). Modeling and stability analysis of a dfig-based windpower generator interfaced with a series-compensated line. IEEE Transactions on Power Delivery, 24(3), 1504-1514.

13. Preece, R., Woolley, N. C., \& Milanović, J. V. (2013). The probabilistic collocation method for powersystem damping and voltage collapse studies in the presence of uncertainties. IEEE Transactions on Power Systems, 28(3), 2253-2262.

14. Tatang, M. A. (1995). Direct incorporation of uncertainty in chemical and environmental engineering systems. Massachusetts Institute of Technology. 